\title{
The Orientation of Elliptical Galaxy NGC 741
}

\author{
A. K. Diwakar \\ Department of Physics, Institute for Technology and Management University Raipur, Chhattisgarh, India \\ E-mail: \\ Corresponding Author: diw.arun@gmail.com, Tel.: +91-98279-37147
}

Available online at: www.isroset.org

Received $21^{\text {st }}$ Aug, 11 Sep 2017, 2017, Accepted $19^{\text {th }}$ Oct 2017, Online $30^{\text {th }}$ Oct 2017

\begin{abstract}
Determination of the orientation of the elliptical galaxies is an important problem. We determine the orientation of the light distribution of individual elliptical galaxies by combining the profiles of photometric data from the literature with triaxial models. The likelihood of obtaining the data from a model is a function of the parameters describing the intrinsic shape and the orientation. Integrating the likelihood over the shape parameters, we obtain the estimates of the orientation. Apply the methodology to determine the orientation of the galaxy NGC 741.
\end{abstract}

Keywords - Galaxy-Photometry, Galaxy-Orientation

\section{INTRODUCTION}

The orientation of the individual elliptical galaxies have been investigated by [1], [2], [3] and [4].The methodology is based on Bayesian statistics, and is described in [5], [6] and more recently in [7]. The likelihood of obtaining the data from a model in a chosen orientation is a function of the view angle and the shape parameters. The likelihood is multiplied by a model for the parent distribution of these parameters (the prior), and integrated over the parameters that one is not trying to constrain. The normalized result is the posterior probability distribution for the parameters of uninteresting. Probability of the shape is obtained by integrating over the uninteresting orientation parameter.

Section II presents the triaxial models to find the orientation of the elliptical galaxy NGC 741, Section III presents the methodology to determine the orientation, Section IV presents the observational data of the galaxy, Section V presents the calculation and statistical summary of the orientation of NGC 741, Section VI describe the Result and discussion.

\section{MODELS}

\section{A. $\mathbf{M}^{2}$ Models}

A triaxial generalization of [8] spherical density distribution $\rho(r)$ is made triaxial by considering the distribution $\rho(r)$ and replacing $r$ by $M$, and its modify by [6],

$$
\rho\left(M^{2}\right)=\frac{M_{0}(3-\gamma) b}{4 \pi P Q} M^{-\gamma}(b+M)^{-4+\gamma}
$$

where $M^{2}=x^{2}+\frac{y^{2}}{P}+\frac{z^{2}}{P}$. Here, $(x, y, z)$ are the usual Cartesian coordinates, $r$ is the spherical radial coordinate and

$$
\begin{aligned}
& \mathrm{P}^{2}(M)=\frac{\beta b^{2} p_{0}^{-2}+M^{2} p_{\infty}^{-2}}{\beta b^{2}+M^{2}} \\
& \mathrm{Q}^{2}(M)=\frac{\beta b^{2} q_{0}^{-2}+M^{2} q_{\infty}^{-2}}{\beta b^{2}+M^{2}}
\end{aligned}
$$

The constant density surfaces are coaxial and coaligned ellipsoids with varying axial ratios $(P, Q)$, which are $\left(p_{0}, q_{0}\right)$ at small radii and are $\left(p_{\infty}, q_{\infty}\right)$ at large radii. In above, $b$ is the scale length and $\beta>0$ is a parameter. The parameter $\beta$ alters the values of $(P, Q)$ in the intermediate region. These models are presented in [4] and [6].

\section{$B$. fgh Models}

Another form of triaxial generalization of [8] model is presented in [9] and its modified by [6]. Rewriting $\rho(r)$ as $f(r)$, the triaxial model is density distribution of the form

$$
\rho=f(r)-\left[g(r)+g_{1}(r)\right] Y_{2}^{0}+\left[h(r)+h_{1}(r)\right] Y_{2}^{2}
$$

where $\rho$ is the density in the usual spherical polar coordinates $(r, \theta, \varphi), \mathrm{g}(\mathrm{r})$ and $\mathrm{h}(\mathrm{r})$ are two suitably chosen radial functions, $Y_{2}^{0}=\frac{3}{2} \cos ^{2} \theta-\frac{1}{2}$ and $Y_{2}^{2}=3 \sin ^{2} \theta \cos 2 \varphi$ are the usual spherical harmonics and

$$
\begin{aligned}
& g_{1}(r)=\alpha \frac{L r_{1}}{4 \pi\left(r+r_{2}\right)} 30 r r_{1} \\
& h_{1}(r)=\alpha \frac{L r_{3}}{4 \pi\left(r+r_{4}\right)} 30 r r_{4}
\end{aligned}
$$


In above, $g(r), h(r)$ and the parameters $r_{1} . . r_{4}$ are the same as in [9]. $g_{1}(r)$ and $h_{1}(r)$ are introduced in [6], which for choices of parameter $\alpha>0$, alter the profiles of $\varepsilon$ and $\theta$ in the intermediate region.

Orientation of the elliptical galaxies can be determined using these models exhibit ellipticity variation and position angle twist. They use ellipticities $\epsilon_{\text {in }}, \epsilon_{\text {out }}$ and the position angle difference $\Theta_{\text {out }}-\Theta_{\text {in }}$ at two suitably chosen points $R_{\text {in }}$ and $\mathrm{R}_{\text {out }}$ from the profiles of the photometric data of the galaxies. The orientation is described by the parameters of the orientation as a function of polar coordinates $\left(\theta^{\prime}, \varphi^{\prime}\right)$ of the line of sight. $\left(\mathrm{q}_{0}, \mathrm{q}_{\infty}, \mathrm{T}_{0}, \mathrm{~T}_{\infty}\right)$, where $\mathrm{q}_{0}, \mathrm{q}_{\infty}\left(\mathrm{T}_{0}, \mathrm{~T}_{\infty}\right)$ are short to long axial ratios (triaxialities) at small and at large radii, respectively. They find that the best constrained shapes parameters are the axial ratios $\mathrm{q}_{0}, \mathrm{q}_{\infty}$ and the absolute value of the triaxial difference $\mathrm{T}_{\mathrm{d}}$ defined as $\left|\mathrm{T}_{\mathrm{d}}\right|=\left|\mathrm{T}_{\infty}-\mathrm{T}_{0}\right|$.

The orientation is given by a Bayesian probability distribution. The likelihood of obtaining the data from a model is a function of the parameters describing the orientation. Integrating the likelihood over the uninterested shape parameters, we obtain the estimates of the orientation. We find that the position angle difference between the two suitable chosen points from the profiles of the photometric data plays a key role in constraining the orientation of the galaxy.

\section{ORIENTATION OF NGC 741}

The observed data of NGC 741 is $\epsilon_{\text {in }}=0.129, \epsilon_{\text {out }}=0.188, \theta_{d}$ $=2.5^{\circ}$ at $R_{\text {in }}=6.8 \operatorname{arcsec}$ and $R_{\text {out }}=16.5$ arcsec. Effective radius $R_{e}$ of $N G C 741$ is 57.5 arcsec. This data is obtained from R-band photometry of [10]. While applying this data to calculate the probability of the intrinsic shape, we consider the uncertainty of 0.02 in ellipticities, and the uncertainty of $1^{\mathrm{O}}$ in position angles both at $\mathrm{R}_{\mathrm{in}}$ and $\mathrm{R}_{\text {out }}$. We use the ensemble of models, as described in section II, $\mathbf{M}^{2}$ models with $\beta=5.0,2.5,1.0$, and fgh models with $\varepsilon=0.0,2.5,5.0$. Integrating the marginal posterior density over all possible values of $\mathrm{q}_{0}$ and $\mathrm{q}_{\infty}$, and taking unweighted sum over all these models, we obtained orientation estimate the probability $\mathrm{P}$ as a function of $\left(\theta^{\prime}, \varphi^{\prime}\right)$ is a model independent.

\section{RESUltS AND DISCUSSION}

Table 1. presents the statistical summary of orientation of the galaxy NGC 741. The determination of the orientation of this galaxy is present in fig. 1. The probability of the orientation plotted in dark grey shade: darker is the shade, higher is the probability. The white contour encloses the region of $68 \%$ highest posterior density, which may be interpreted as $1 \sigma$ error bar. The plus mark is the true orientation of the galaxy.
We find a good agreement between the results of probability calculations and the true orientation.

Table 1. Statistical summary of the orientation of NGC 741.

\begin{tabular}{|c|c|c|c|c|c|c|}
\hline \multicolumn{2}{|c|}{ Models } & \multirow{2}{*}{$\begin{array}{c}\boldsymbol{\theta}_{\mathbf{p}}^{\prime} \\
67.5\end{array}$} & \multirow{2}{*}{$\begin{array}{c}\varphi_{\mathbf{p}}^{\prime} \\
49.5\end{array}$} & \multirow{2}{*}{$\begin{array}{l}\left\langle\boldsymbol{\theta}^{\prime}\right\rangle \\
52.6\end{array}$} & \multirow{2}{*}{$\begin{array}{l}\left\langle\varphi^{\prime}\right\rangle \\
89.2\end{array}$} & \multirow{2}{*}{ 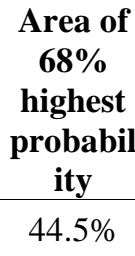 } \\
\hline \multirow{3}{*}{$\mathbf{M}^{2}$} & $\beta=1.0$ & & & & & \\
\hline & $\beta=2.5$ & 73.5 & 49.5 & 51.6 & 88.8 & $48.9 \%$ \\
\hline & $\beta=5.0$ & 73.5 & 19.5 & 48.4 & 89.8 & $53.5 \%$ \\
\hline \multirow{3}{*}{ fgh } & $\varepsilon=0.0$ & 79.5 & 25.5 & 53.1 & 84.1 & $49.0 \%$ \\
\hline & $\varepsilon=2.5$ & 85.5 & 28.5 & 47.3 & 87.0 & $56.4 \%$ \\
\hline & $\varepsilon=5.0$ & 40.5 & 20.5 & 43.9 & 87.35 & $59.2 \%$ \\
\hline \multicolumn{2}{|c|}{$\begin{array}{l}\text { Sum over } \\
\text { all models }\end{array}$} & 79.5 & 22.5 & 49.5 & 87.7 & $55.1 \%$ \\
\hline
\end{tabular}

The plot of the posterior probability distribution $\mathrm{P}$ of the orientation as a function of polar coordinates $\left(\theta^{\prime}, \varphi^{\prime}\right)$ of the line of sight as shown in fig. 1 . We use a rectangular plot with $\theta^{\prime}$ going from $0^{\circ}$ to $90^{\circ}$ in the vertical direction from bottom to top and $\varphi^{\prime}$ going from $0^{\circ}$ to $180^{\circ}$ in the horizontal direction from left to right. The observational data use from [10] and use the $M^{2}$ model with $\beta=1.0,2.5,5.0$ and $f g h$ model with $\alpha=0.0,2.5,5.0$.

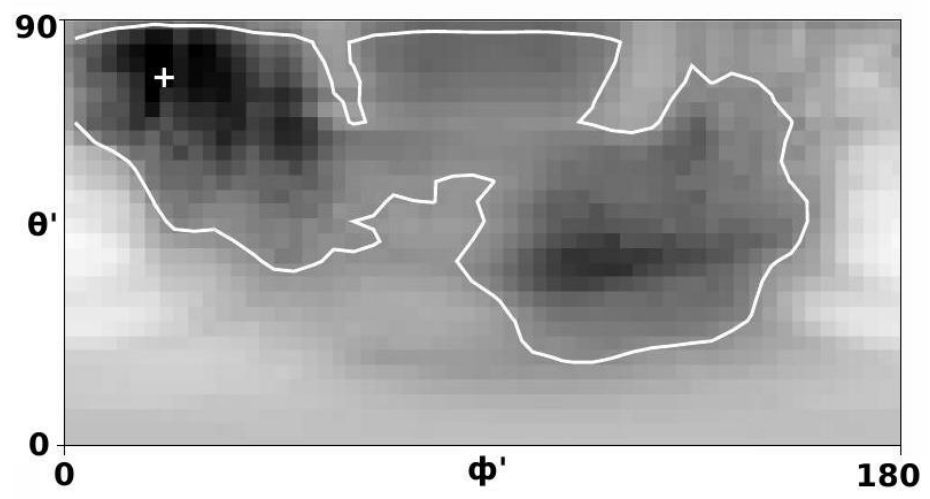

Figure 1. Plot of the posterior probability distribution $\mathrm{P}$ as a function of $\left(\theta^{\prime}, \varphi^{\prime}\right)$ for NGC 741 . Plus marks the location of the maximum probability.

\section{ACKNOWLEDGMENT}

I would like to thank the ITM University Raipur, for the technical supports. 


\section{REFERENCES}

[1] J. Binney, "Testing for triaxiality with kinematic data", Monthly Notices of the Royal Astronomical Society, Vol. 212, pp 767-781, 1985 .

[2] T.S. Statler, "Upcovering the intrinsic shapes of elliptical galaxies. III practical modeling, The Astronomical Journal, Vol.425, pp. 500-529, 1994.

[3] J. Bak, T.S. Statler, "The Intrinsic Shape Distribution of a Sample of Elliptical Galaxies", The Astronomical Journal, Vol. 120, pp. 110122, 2000.

[4] D.K. Chakraborty, "Ellipsoidal mass models with varying axial ratios" Astronomy \& Astrophysics,,Vol. 423, pp. 501-505, 2004.

[5] D.K. Chakraborty, A.K. Diwakar, "The orientation of elliptical galaxies', Astrophysics and Space Science, Vol. 331, pp. 419-428, 2010.

[6] D.K. Chakraborty, A.K. Diwakar, S.K. Pandey, "Intrinsic shape of very flat elliptical galaxies", Monthly Notices of the Royal Astronomical Society, vol. 412, pp. 585-590, 2010.

[7] A.K. Diwakar, "Intrinsic shape of elliptical galaxy NGC 471", International Journal of Trend in Research and Development, Volume 4(4), pp. 382, 2017.

[8] W. Dehnen, "A family of potential-density pairs for spherical galaxies and bulges", Monthly Notices of the Royal Astronomical Society, Vol. 265, pp. 250-256, 1993.

[9] P.T. de Zeeuw, C.M. Carollo, "A family of triaxial mass models with central cups", Monthly Notices of the Royal Astronomical Society, Vol. 281, pp. 1333-1340, 1996.

[10] R.F. Peletier, R.L. Davies, G.D. Illingworth, L.E. Davis, M. Cawson, "CCD surface photometry of galaxies with dynamical data IL UBR Photometry of 39 elliptical galaxies", The Astronomical Journal, Vol. 100, pp. 1091-1142, 1990.

\section{AUTHORS PROFILE}

A. K. Diwakar, Ph.D. in Physical Science from Pt. Ravishankar Shukla University, Raipur, C.G. in 2010. He is currently working as Assistant Professor in Department of Physics, Institute for Technology and Management University Raipur, C.G. India, since 2013. He is 2 year teaching 2011-13, in the Department of Physics and Astrophysics, Pt. Ravishankar Shukla University, Raipur, C.G. India. He is

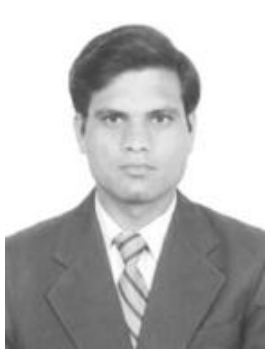
a Life member of Astronomical Society of India, since 2011. He has published good research papers in reputed international journals including Thomson Reuters, SCI and conferences and it's also available online. His main research work focuses on elliptical galaxies, modelling of triaxial mass models, and Astronomy and Astrophysics. He has 7.5 years of teaching experience and research experience. 